\title{
Auditory discrimination learning: Prototype storage and distinctive features detection mechanisms
}

\author{
EDWIN G. AIKEN ${ }^{2}$ \\ NA VAL PERSONNEL RESEARCH ACTIVITY, SAN DIEGO
}

\begin{abstract}
The relative contributions of prototype storage and distinctive features detection mechanisms hypothesized to be operating in successive discrimination learning were assessed by the extent of negative transfer between auditory discrimination tasks. The results indicated that both mechanisms were involved, but that prototype storage was the dominant process. This outcome is compared with previous research on tactual and visual discrimination leaming. Experimental variables are suggested that might control which mechanism predominates.
\end{abstract}

Recently (Pick, 1965) presented data suggesting that two different mechanisms are operating in visual and tactual discrimination learning. Pick calls the first mechanism Schema Learning. This refers to an increasingly accurate memory storage of all the properties of the standard stimuli in a discrimination learning task. These stored standards are sometimes referred to as prototypes, which facilitate "same" or "different" judgments of comparison stimuli. Pick refers to the second mechanism as Distinctive Features. According to this principle, improvement in discrimination consists in $\mathrm{S}$ detecting those stimulus dimensions that are critical to judging accurately the standard and comparison stimuli as "same" or "different." This hypothesized mechanism is said by Pick to derive from the theorizing of the Gibsons (Gibson \& Gibson, 1955.)

One way to test for the operation of one or both mechanisms in any particular discrimination learning situation is through a transfer design. If Ss store prototypes of the standard stimuli, changing them should produce a decrement in performance. If Ss learn only the features of the stimuli that distinguish them, then changing these features should produce a performance decrement. For purposes of this report, the two mechanisms will be referred to as Prototype Storage and Distinctive Features Detection.

Pick interprets her data on visual and tactual discrimination learning with children as indicating the dominance of distinctive features detection. Prototype Storage is interpreted as having a subsidiary function, in successive discrimination learning only, where the separation of the standard and comparison stimuli places a memory requirement on $S$.

The present investigation tests the hypothesis that Prototype Storage is the dominant mechanism in successive auditory discrimination learning with adult Ss.

\section{Subjects}

\section{METHOD}

Forty-eight Navy recruits with normal hearing were used as Ss. Data were obtained on four Ss at a time.

\section{paratus and Task Stimuli}

An oscillator output was modified to permit the rapid selection by $E$ of one of four standard signal frequencies. A series of calibrated variable resistors allowed $E$ to select one of four signal amplitudes, and an interval timer permitted $\mathrm{E}$ to select one of four signal durations. Finally, standard signal directionality was controlled by slight attenuation of the signal to one or the other side of Koss Pro-4 stereo headsets. In a similar fashion, E could select the frequency, amplitude, duration, and directionality of the comparison signal. The standard signals were separated from the comparison signals by $1 \frac{1 / 2}{\mathrm{sec}}$ and the fime between the termination of one signal pair and the onset of the next was $25 \mathrm{sec}$. Table 1 presents the acoustical properties of the four standard signals along with the variations in the dimensions of the comparison signals used with each. The particular comparison signal values were selected on the basis of informal pretests that indicated that they were generally of equal difficulty from une standard to another and from one dimension to another.

\section{Procedure}

Training. All $48 \mathrm{~S}$ s were trained in the same way. Ilowever, to counterbalance any differences in difficulty of paticular combinations of standards and comparison signal thanges, the Ss were divided into four groups of $12 \mathrm{Ss}$ each. The groups were distinguished by the particular combination of tramaml signals and comparison signal dimension differences te whict they were exposed. The combinations were: (a) Standard Signals $\mathrm{A}$ and $\mathrm{C}$, with comparison signal differences in the frequency or durtion

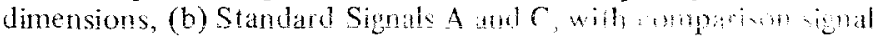

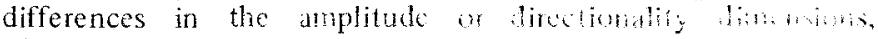
(c) Standard Signals B and D, with comparison igm Jerrates in the frequency or duration dimensions, and (d) Standard Signals $B$ and D, with comparison signal differences in the amplitude or directionality dimensions.

The training of all Ss consisted in requiring a same or different judgment on 96 signal pairs. Ss indicated their judgments by closing one of two switches on a response panel within $5 \mathrm{sec}$ of the termination of the comparison signal. Informative feedback was provided after every judgment by means of lights aligned with the correct answers. On 48 of the signal pairs one standard was presented and on the other 48 pairs the other was presented. On 24 of the pairs with each standard, the comparison signal was the standard signal replayed, and on the other 24 with each standard one of the two comparison signal dimensions was different, 12 times for each dimension. The 96 signal pairs were

Table 1

Acoustical Properties of Each Standard Signal and Their Possible Comparison Signal Changes

\begin{tabular}{lcccc}
\hline \multicolumn{1}{c}{ Signals } & Frequency $(\mathrm{Hz})$ & Amplitude $(\mathrm{dBm})^{\mathrm{a}}$ & Duration (sec) \\
\hline Standard A & 250 & -10 & 1.00 & Direction \\
Comparison A & 254 & -8 & 1.50 & Left \\
Standard B & 400 & -15 & 2.00 & Right \\
Comparison B & 408 & -12 & 2.50 & Left \\
Standard C & 550 & -20 & 3.00 & Right \\
Comparison C & 560 & -17 & 4.00 & Left \\
Standard D & 700 & -25 & 5.00 & Left \\
Comparison D & 712 & -22 & Right \\
\hline
\end{tabular}

$a \mathrm{OdBm}=1 \mathrm{mw}$ through $a 600 \mathrm{ohm}$ resistance 
Table 2

Errors on the Transfer Test

\begin{tabular}{lccccc}
\hline & \multicolumn{5}{c}{ Dimensions of Comparison Signal Changes } \\
\cline { 2 - 6 } Transfer & Frequency or Duration & Amplitude or Direction \\
\cline { 2 - 6 } Group & A \& C & B \& D & A \& C & B \& D & Total \\
\hline & 18 & 23 & 34 & 20 & 95 \\
E-1 & 19 & 22 & 23 & 9 & 73 \\
E-2 & 40 & 27 & 29 & 28 & 124 \\
C & 77 & 72 & 86 & 57 & 292 \\
Total & &
\end{tabular}

Note: Column headings indicate the particular combination of standards and comparison signal changes used in the transfer test.

$N=4$ in each subgroup.

randomly ordered and the Ss were informed that half the pairs would be the same and half different. Counters connected to a voltage comparator circuit totaled the number of correct responses for each $\mathrm{S}$.

Transfer. The $48 \mathrm{Ss}$ were divided into three transfer conditions. In Experimental Group 1 (E-1), the signal pairs during transfer were designed to optimize interference if Prototype Storage had taken place during training, i.e., the standard signals were changed to those not used during training, while the two dimensions of comparison signal differences remained the same. In Experimental Group 2 (E-2) the signal pairs during transfer were designed to optimize interference if Distinctive Features Detection had taken place during training, i.e., the two dimensions of comparison signal differences experienced during training were changed, while the standard signals remained the same. The control group (C) was designed to provide a baseline from which to assess the relative transfer decrements of E-1 and E-2, i.e., both the standard signals and the dimensions of comparison signal differences were changed from what they had been during training. The counterbalancing used during training required that each of the transfer groups have four subgroups (see Table 2).

The transfer test contained 24 signal pairs presented in a random order. Ss were again informed that half of the pairs would be the same and half different, and informative feedback continued to be provided after each judgment.

\section{Training}

\section{RESULTS AND DISCUSSION}

As a check on the initial equality of the performance during training of the three groups subsequently given differential treatment during the transfer test, the number of errors during training for the three transfer groups was submitted to an analysis of variance. The $F$ obtained fell far short of significance.

\section{Transfer}

Table 2 presents the number of errors made by each transfer group. A 4 by 3 repeated-measures analysis of variance was performed on these data. The four-level variable was the standard signal, comparison signal combinations used during transfer, and the three-level variable was the transfer conditions. The $F$ for signal combinations was 9.04 , which for $3 / 36 \mathrm{df}$ is significant beyond the .001 level of confidence. This simply reflects the fact that certain signal combinations produced more interference during transfer than others. The variance source of central interest is that associated with the transfer conditions. The $F$ obtained was 30.15 , which for $2 / 36 \mathrm{df}$ is significant beyond the .001 level. The mean number of errors on the 24 signal transfer test for each group was: 5.94 for E-1, 4.56 for E-2, and 7.75 for C. A Newman-Keuls test for the multiple comparison of means indicated that the $C$ group made significantly more errors $(p<.01)$ than either E-1 or E-2, and E-1 made significantly more errors than $E-2(p<.01)$. The interaction of signal combination and transfer condition was also significant beyond the .001 level $(F=7.35$, df $=6 / 36)$. This reflects the fact that certain changes from training to transfer presented more difficulty to one training group than another (see Table 2).

On the basis of these data, it would appear that both Prototype Storage and Distinctive Features Detection mechanisms are involved in auditory discrimination learning, with Prototype Storage dominant.

These results accord with the assumption that successive stimulus presentation will favor the storage of prototypes because of the memory requirement presented to $S$ by the separation interval between the standard and comparison stimuli. Further evidence on this point has recently been reported by Manatis (1968). He inferred from his data that requiring $S$ to later recognize rather than immediately discriminate visual forms resulted in more effective perceptual storage. On the other hand, in no case did Pick (1965) find changing standards more detrimental than changing distinctive features. This conflict with the present data may simply reflect the different modalities and/or age of the Ss involved in the studies. However, two other explanations are possible. First, in Pick's studies the number of comparisons in which "different" was the correct response was always greater than "same," which may have biased Ss to search for distinctive features. Second, in the successive tactual discrimination study reported by Pick it is not clear that the order of stimulus contact was always the same from pair to pair. If it was not, $S$ would find it far more difficult to define the standard for purposes of storage. In any event, when the number of acoustic pairs are equally divided between same and different and the order of signal exposure controlled, the hypothesized prototype storage mechanism appears to predominate over the hypothesized distinctive features detection mechanism.

Several variables appear promising for further research in this area. The most obvious is the interstimulus interval. Longer intervals should force greater and greater dependence upon Prototype Storage. Variables from the theory of signal detectability (Swets, Tanner, \& Birdsall, 1961) might also be helpful in elucidating when discrimination learning is dominated by one or the other mechanism. For example, as suggested earlier, one might vary the a priori probabilities of same and different pairs during training; the hypothesis being that a predominance of same pairs would favor Prototype Storage and a predominance of different pairs would favor Distinctive Features Detection. Similarly, one might vary the value associated with correctly identifying same or different pairs, and thereby bias the learner to one mechanism or the other.

\section{REFERENCES}

GIBSON, J. J., \& GIBSON, E. J. Perceptual learning: Differentiation or enrichment? Psychological Review, 1955, 62, 3241.

MANATIS, J. V. Transfer effects in perceptual learning: A comparison of recognition and discrimination. Paper read at the meeting of the Southern Society for Philosophy and Psychology, April 1968.

PICK, A. D. Improvement of visual and tactual form discrimination. Journal of Experimental Psychology, 1965, 69, 331-339.

SWETS, J. A., TANNER, W. P., Jr., \& BIRDSALL, T. G. Decision processes on perception. Psychological Review, 1961, 68, 301-340.

\section{NOTES}

1. The opinions and assertions contained herein are those of the writer and are not to be construed as official or as reflecting the views of the Navy Department or naval service.

2. Address: Naval Personnel Research Activity, San Diego, California 92152.

(Accepted for publication January 20, 1969.) 